\title{
Anderson localization of electron states in graphene in different types of disorder
}

\author{
Shi-Jie Xiong* \\ National Laboratory of Solid State Microstructures and Department of Physics, \\ Nanjing University, Nanjing 210093, China \\ Ye Xiong \\ College of Physical Science and Technology, \\ Nanjing Normal University, Nanjing 210097, China
}

\begin{abstract}
Anderson localization of electron states on graphene lattice with diagonal and off-diagonal (OD) disorder in the absence of magnetic field is investigated by using the standard finite-size scaling analysis. In the presence of diagonal disorder all states are localized as predicted by the scaling theory for two-dimensional systems. In the case of OD disorder, the states at the Dirac point $(E=0)$ are shown to be delocalized due to the specific chiral symmetry, although other states $(E \neq 0)$ are still localized. In OD disorder the conductance at $E=0$ in an $M \times L$ rectangular system at the thermodynamical limit is calculated with the transfer-matrix technique for various values of ratio $M / L$ and different types of distribution functions of the OD elements $t_{n n^{\prime}}$. It is found that if all the $t_{n n^{\prime}}$ 's are positive the conductance is independent of $L / M$ as restricted by 2 delocalized channels at $E=0$. If the distribution function includes the sign randomness of elements $t_{n n^{\prime}}$, the conductivity, rather than the conductance, becomes $L / M$ independent. The calculated value of the conductivity is around $\frac{4 e^{2}}{h}$, in consistence with the experiments.
\end{abstract}

PACS numbers: $72.80 . \mathrm{Ng}, 73.63 .-\mathrm{b}, 81.05 . \mathrm{Uw}, 73.23 .-\mathrm{b}$

*Electronic address: sjxiong@nju.edu.cn 


\section{INTRODUCTION}

As a potential candidate material for carbon-based electronics, graphene, a twodimensional (2D) honeycomb lattice of carbon atoms, has attracted much attention $[1,2,3,4,5]$. It is found that both the type (electrons or holes) and the number of carriers can be tuned by changing the gate voltage [6, 7]. Such tunability originates from the specific dispersion relation of the massless Dirac fermions in graphene [8, 9]. Its transport properties are very special. There exists a universal maximal resistivity for all samples with the Fermi level near the Dirac point, independent of their shapes and mobility. The maximal resistivity [3] implies the existence of channels of extended states at the thermodynamical limit, and the shape independence of the resistivity suggests that both the number and spatial configuration of these extended channels depend on the shape. This behavior is certainly exotic as from the scaling theory all the states are localized in 2D systems [10]. The non-zero minimal conductivity has been investigated by several theoretical studies using different methods [11, 12, 13, 14, 15]. The minimum conductivity was correctly demonstrated, but as to the reason for it and as to its exact value being $4 e^{2} / h$ or $4 e^{2} / \pi h$ there is no consensus at present. Morozov et al. have found that in graphene the weak localization is strongly suppressed and they attributed this to mesoscopic corrugations of graphene sheets which can cause a de-

phasing effect similar to that of a random magnetic field [16]. Theoretically, the influence of disorder on 2D electron gases on the honeycomb lattice was studied with a self-consistent Born approximation [17, 18]. Pereira et al. have investigated the localized states near vacancies in graphene [19]. Khveshchenko has carried out an analysis of quantum interference effects in disordered graphene and specified the conditions for the quantum correction to the conductivity to be positive, negative, and zero [20]. By evaluating the dependence of the magnetoresistance of graphene on the relaxation rates from different sources McCann et al. showed that the warping disorder and the disorder due to atomically sharp scatterers have different effects on the magnetoresistance [21]. Morpurgo and Guinea investigated the effects of static disorder caused by curvature or topological defects and found that when the intervalley scattering time is long enough an effective time-reversal symmetry breaking can be induced [22]. Aleiner and Efetov showed two different types of logarithmic contributions to conductivity by describing the disorder with a renormalization-group equation [23]. From a combination of mean-field and bosonization methods Altland pointed out that there are 
several mechanisms conspiring to protect the conductivity although disordered graphene is subject to the common mechanisms of Anderson localization [24]. From the linear response theory Ziegler indicated that the transport properties in graphene are dominated by diffusion [25]. Based on numerical study with finite-size Kubo formula for doped graphene, Nomura and MacDonald found distinct differences between the cases of short-range and Coulomb randomly distributed scatterers and speculated that the transport properties in graphene are related to the Boltzmann theory for Coulomb scatterers [26]. Sheng et al. carried out transfer matrix studies of graphene in a magnetic field to investigate the disorder effect and phase diagram [27]. Louis et al. calculated conductances for samples with edge disorder and obtained size independent conductivity as $\sim 4 e^{2} / \pi h$ at $E=0$ [28]. Moreover, by adopting disorder model without intervalley scattering, Ostrovsky et al. constructed an effective field theory and showed that the system is at a quantum critical point with a universal value of the conductivity of the order of $e^{2} / h$ [29]. With a similar model Titov demonstrated the impurity-assisted tunneling by using the transfer matrix approach [30]. Bardarson et al. numerically showed that the conductivity at the Dirac point increases logarithmically with sample size in the absence of intervalley scattering [31]. From numerical calculation Nomura et al. demonstrated that all states are delocalized even in the strong disorder regime if the intervalley scattering is excluded [32]. San-Jose et al. investigated the full transport statistics of graphene at low dopings without intervalley scattering and found identical scaling of all current cumulants [33].

In this paper we investigate the Anderson localization of fermions at the Dirac point in graphene with different types of disorder by using the standard finite-scaling analysis together with the transfer-matrix technique. The Dirac point corresponds to the band center $(E=0)$ of a tight-binding Hamiltonian on the honeycomb lattice. In the real-space tight-binding representation the disorder can be introduced with randomly distributed site energies, as called diagonal disorder, or with random hopping integrals named as off-diagonal (OD) disorder. It is known that the localization behavior at the band center of a bipartite lattice with OD disorder is anomalous due to the chiral symmetry [34, 35, 36]. For instance, the decay of wavefunctions at the band center on 2D square lattice with $\mathrm{OD}$ disorder is much weaker than exponential. The honeycomb lattice is also bipartite, and there are other unusual features at the Dirac point such as the massless Dirac fermion dispersion relation. So it is interesting to investigate the difference in the localization behavior at the Dirac point 
between diagonal and off-diagonal disorders. We derive the formula of transfer matrix for honeycomb lattice from which the numerical studies can be carried out to calculate interesting properties such as the rescaled localization length, the size and shape dependencies of the conductance. The finite-size scaling analysis reveals that although all the states are localized in the system with diagonal disorder, in the case of OD disorder, the states at the Dirac point are delocalized. From the calculated shape dependence of the conductance at $E=0$ on an $L \times M$ rectangular lattice we find that when all the hopping integrals are positive, the conductance is independent of $L / M$, but if the distribution includes both the positive and negative hopping integrals, the conductivity becomes shape independent. Diagonal disorder corresponds to atomic scatterers such as doped impurities, while OD disorder without sign changes of hopping integrals represents randomness of bond lengths caused by topological corrugations or warping in the sheets. For graphene OD disorder is more important than diagonal disorder owing to the ultrathin one-layer structure and the purity requirement in preparing the samples. In both types short-range disorder can exist and the intervalley scattering is not excluded. Thus the obtained results should be different from those by using models without intervalley scattering due to different symmetry classes. The sign randomness of hopping integrals can be produced by geometric or Berry phase $\sim \pm \pi$ which is acquired by electron wavefunctions in every period of cyclic evolution around Dirac point in parameter space. The Dirac point can serve as the degenerate point for geometric phase in "slow" cyclic evolutions, such as in-plane vibrations of displacement between two sublattices, due to the specific dispersion relation and electron-lattice interaction.

\section{BASIC MODEL AND FORMALISM}

The main features of the band structure in graphene can be well described by a tightbinding Hamiltonian with one $\pi$ orbital per site on a honeycomb lattice [8]:

$$
H=\sum_{n} \epsilon_{n} a_{n}^{\dagger} a_{n} \sum_{\left\langle n, n^{\prime}\right\rangle}+t_{n n^{\prime}}\left(a_{n}^{\dagger} a_{n^{\prime}}+a_{n^{\prime}}^{\dagger} a_{n}\right)
$$

where $a_{n}^{\dagger}\left(a_{n}\right)$ creates (annihilates) an electron at site $n, \epsilon_{n}$ is the energy level at site $n,\langle\ldots\rangle$ denotes the nearest-neighbor $(\mathrm{NN})$ sites, and $t_{n n^{\prime}}$ is hopping integral between NN sites $n$ and $n^{\prime}$. Here, the spin indices are not explicitly included. In the absence of disorder, $\epsilon_{n}=0$ for all $n$ and $t_{n n^{\prime}}=t_{0}$ for all $\left\langle n n^{\prime}\right\rangle$, this Hamiltonian leads to the 4-component Dirac fermion 
dispersion relation near the Dirac point $E=0$. The diagonal and off-diagonal disorder can be introduced by adopting random variables $\epsilon_{n}$ and $t_{n n^{\prime}}$ satisfying distribution probabilities $P_{d}\left(\epsilon_{n}\right)$ and $P_{o}\left(t_{n n^{\prime}}\right)$, respectively. These probability functions define strengths and types of disorder in the system. Diagonal disorder represents potential fluctuations due to impurities or due to randomness on substrate surface. A most commonly used distribution probability for diagonal disorder is the square function

$$
P_{d}\left(\epsilon_{n}\right)=\left\{\begin{array}{l}
1 / W, \text { for }-W / 2 \leq \epsilon_{n} \leq W / 2, \\
0, \text { otherwise }
\end{array}\right.
$$

where $W$ is the distribution width describing the strength of disorder. For OD disorder, there are different distribution functions describing different types of randomness. If we consider slight fluctuations of bond lengths around their average value due to lattice distortions, the following distribution is suitable

$$
P_{o 1}\left(t_{n n^{\prime}}\right)=\left\{\begin{array}{l}
1 / \lambda, \text { for } t_{0}-\lambda / 2 \leq t_{n n^{\prime}} \leq t_{0}+\lambda / 2 \\
0, \text { otherwise }
\end{array}\right.
$$

where $\lambda\left(<2\left|t_{0}\right|\right)$ is a measure of the bond-length fluctuations which make the NN hopping integrals randomly shift from $t_{0}$.

In $P_{o 1}\left(t_{n n^{\prime}}\right)$ all hopping integrals have the same sign as $t_{0}$, reflecting that only changes of magnitude of $t_{n n^{\prime}}$ are considered. In some cases, however, $t_{n n^{\prime}}$ not only fluctuate in magnitude, but also change in sign due to specific physical mechanisms. One of these possible mechanisms is the geometric phase due to dynamical vibrations. The on-plane displacement between two sublattices, denoted as $\mathbf{d}=\left(d_{x}, d_{y}\right)$, changes all the NN bond lengths and thus linearly couples with electron states in the regime of small $|\mathbf{d}|$. At a Dirac point $(E=0)$ the states are doubly degenerate at $\mathbf{d}=0$. The displacement $\mathbf{d} \neq 0$ linearly removes the degeneracy, leading to conical energy surfaces in the parameter space spanned by $d_{x}$ and $d_{y}$ with the degenerate point situated at the origin $d_{x}=d_{y}=0$. Owing to thermal excitations or even to zero-point oscillations, $d_{x}$ and $d_{y}$ vibrate and both of them are dependent on time with period $T=2 \pi / \omega$ where $\omega$ is the frequency of vibrations. Usually the amplitudes and relative phase of $d_{x}$ and $d_{y}$ vibrations are statistically random. For generic values of them, the track in the $d_{x}-d_{y}$ parameter space in a period is an ellipse encircling the degenerate point. Thus, according to the Berry theorem, in each period of vibrations electron wavefunctions at Dirac point will acquire a geometric phase $\sim \pm \pi$ in 
addition to the ordinary dynamical phase. At the same time in this period the tunneling electron has traversed a path of length $v_{F} T$ which equals $v_{F} T / l_{0} \mathrm{NN}$ bonds with $v_{F}$ and $l_{0}$ being the Fermi velocity and bond length respectively. This means that the electron states change sign in traversing every $v_{F} T / l_{0}$ bonds. Such an effect occurs very naturally since the vibrations and electron transport are proceeding simultaneously. To include such an effect in a primary way, we introduce the sign-changing probability of an NN hopping $s$. This probability can be estimated from the length by which the electron state changes sign due to the geometric phase, i.e., $s \sim l_{0} /\left(v_{F} T\right)$. With such a sign-change model the wavefunction can automatically change sign in traversing every length of $v_{F} T$ which, averagely, contains only one hopping with opposite sign. This is a special gauge in which the geometric phase acquired in a path of $v_{F} T / l_{0}$ bonds is concentrated into one bond in this path. This gauge is valid under the condition that the interferences between paths shorter than $v_{F} T$ are not relevant. As we will see below, the transport properties at Dirac point in the case of OD disorder (including the sign changes) are scale-invariant. So the short-range processes are not important and the above condition can be satisfied. This sign-change mechanism is different from the dephasing effect in the model of Ref. 16 where the distortions are fluctuating in the space (the ripples) but are static in time. We introduce the following distribution of $t_{n n^{\prime}}$

$$
P_{o}\left(t_{n n^{\prime}}\right)=s P_{o 1}\left(t_{n n^{\prime}}\right)+(1-s) P_{o 1}\left(-t_{n n^{\prime}}\right)
$$

to account for the sign-change probability $s$. When $s=0$ or $s=1$, all the hopping integrals have the same sign and $P_{o}\left(t_{n n^{\prime}}\right)$ reduces to $P_{o 1}\left(t_{n n^{\prime}}\right)$. At $s=0.5$ the probability of the sign changes is maximal. Below we set $t_{0}$ as energy units.

The wavefunction of a fermion can be written as

$$
\psi=\sum_{n} c_{n}|n\rangle
$$

where $c_{n}$ is the amplitude on site $n$. At a given energy $E$ the amplitudes are given by Schrödinger equation $H \psi=E \psi$. The propagation properties of an electron on a finite lattice can be given by a relation between amplitudes on both sides. This is usually formulated by the transfer-matrix method or by the Green function. We note that graphene lattice can be divided into two sublattices as distinguished by red and blue sites in Fig. 1. From the tight-binding nature of the Hamiltonian and the structure shown in Fig. 1 it can be seen that for given $E$ all amplitudes can be iteratively calculated along the $x(y)$ direction if the 


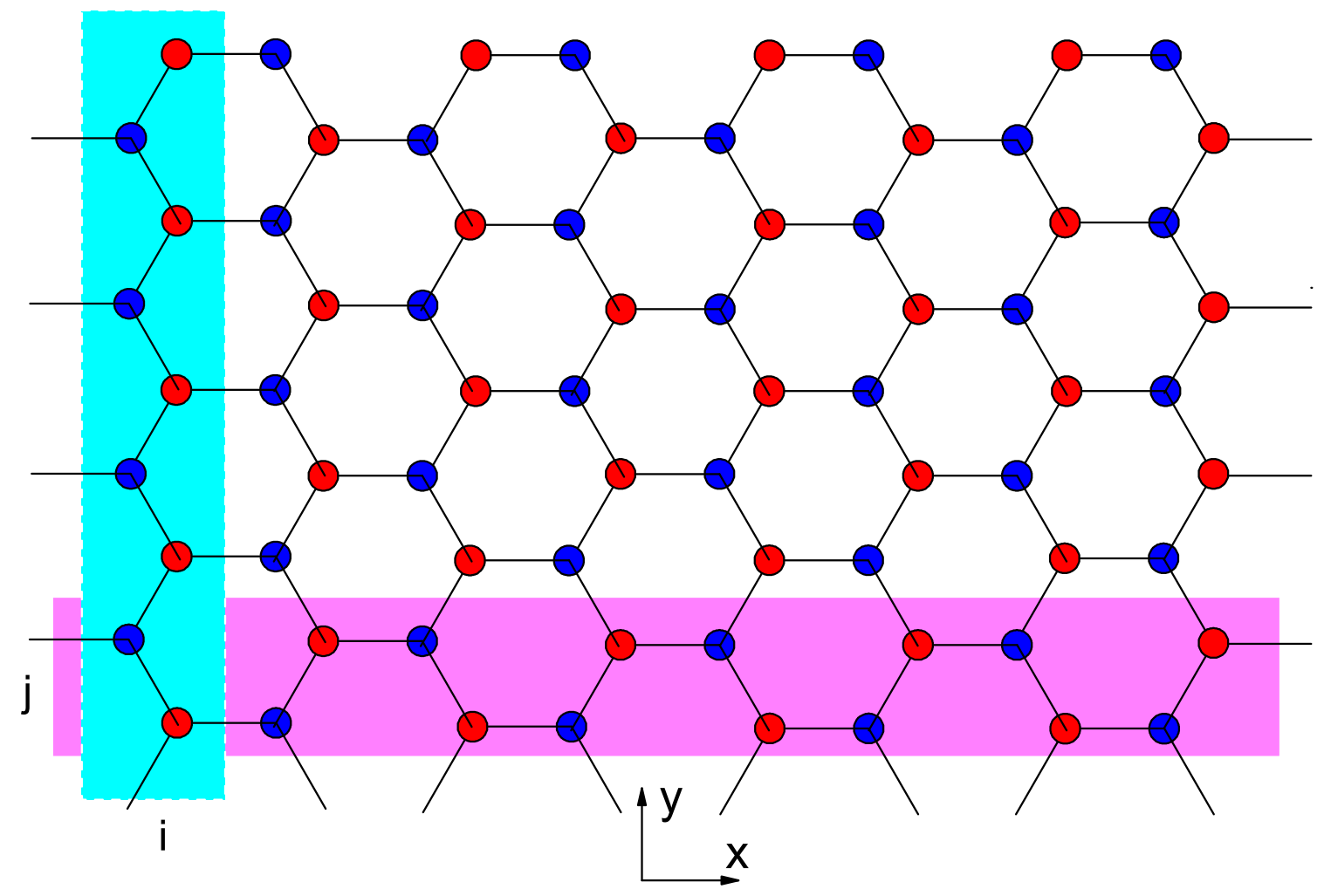

FIG. 1: Structure of graphene lattice. $i$ and $j$ are indices of columns (shaded stripe along the $y$ direction) and rows (shaded stripe along the $x$ direction), and the overlapping area of two stripes is a basic cell where the lower and upper sites correspond to sublattices $l=1$ and $l=-1$, respectively.

amplitudes in the light-cyan shaded column (light-magenta shaded row) are given. From this a transfer matrix along the $x$ or $y$ direction can be given. We identify a site $n$ with three integers, $n \equiv(i, j, l)$, where $i$ and $j$ index the column and row, respectively, and $l= \pm 1$ specifies the sublattice which the site $n$ belongs to. In this notation the iterative relation for amplitudes along the $x$ direction can be derived from Schrödinger equation as

$$
\begin{gathered}
c_{i+1, j,-1}=\frac{\left(E-\epsilon_{i, j, 1}\right) c_{i, j, 1}-t_{i, j, 1 ; i, j,-1} c_{i, j,-1}-t_{i, j, 1 ; i, j+(-1)^{i},-1} c_{i, j+(-1)^{i},-1}}{t_{i, j, 1 ; i+1, j,-1}}, \\
c_{i+1, j, 1}=\frac{\left(E-\epsilon_{i+1, j,-1}\right) c_{i+1, j,-1}-t_{i+1, j,-1 ; i, j, 1} c_{i, j, 1}-t_{i+1, j,-1 ; i+1, j+(-1)^{i}, 1} c_{i+1, j+(-1)^{i}, 1}}{t_{i+1, j,-1 ; i+1, j, 1}},
\end{gathered}
$$

For a system with finite width in the $y$ direction, one can define vectors for columns by $\mathbf{u}_{i, l} \equiv\left(c_{i, 1, l}, c_{i, 2, l}, \ldots, c_{i, M, l}\right)^{T}$, where $M$ is the number of cells in a column. Then Eqs. (6) 
and (7) can be written in a matrix form:

$$
\begin{gathered}
\mathbf{u}_{i+1,-1}=\hat{T}_{1, i} \mathbf{u}_{i, 1}+\hat{T}_{2, i} \mathbf{u}_{i,-1} \\
\mathbf{u}_{i, 1}=\hat{T}_{3, i} \mathbf{u}_{i+1,1}+\hat{T}_{4, i} \mathbf{u}_{i+1,-1}
\end{gathered}
$$

where elements of matrices are given by

$$
\begin{gathered}
\left\{\hat{T}_{1, i}\right\}_{j, j^{\prime}}=\frac{E-\epsilon_{i, j, 1}}{t_{i, j, 1 ; i+1, j,-1}} \delta_{j, j^{\prime}}, \\
\left\{\hat{T}_{2, i}\right\}_{j, j^{\prime}}=-\frac{t_{i, j, 1 ; i, j,-1}}{t_{i, j, 1 ; i+1, j,-1}} \delta_{j, j^{\prime}}-\frac{t_{i, j, 1 ; i, j+(-1)^{i},-1}}{t_{i, j, 1 ; i+1, j,-1}} \delta_{j+(-1)^{i}, j^{\prime}}, \\
\left\{\hat{T}_{3, i}\right\}_{j, j^{\prime}}=-\frac{t_{i+1, j,-1 ; i+1, j, 1}}{t_{i+1, j,-1 ; i, j, 1}} \delta_{j, j^{\prime}}-\frac{t_{i+1, j,-1 ; i+1, j+(-1)^{i}, 1}}{t_{i+1, j,-1 ; i, j, 1}} \delta_{j+(-1)^{i}, j^{\prime}}, \\
\left\{\hat{T}_{4, i}\right\}_{j, j^{\prime}}=\frac{E-\epsilon_{i+1, j,-1}}{t_{i+1, j,-1 ; i, j, 1}} \delta_{j, j^{\prime}} .
\end{gathered}
$$

Here, one may use the periodic or open boundary condition at the ends $j=1$ and $j=M$. We have the iterative relation for whole column

$$
\left(\begin{array}{c}
\mathbf{u}_{i+1,-1} \\
\mathbf{u}_{i+1,1}
\end{array}\right)=\hat{T}_{i}\left(\begin{array}{c}
\mathbf{u}_{i,-1} \\
\mathbf{u}_{i, 1}
\end{array}\right)
$$

where the transfer matrix is

$$
\hat{T}_{i}=\left(\begin{array}{cc}
\hat{T}_{2, i}, & \hat{T}_{1, i} \\
-\hat{T}_{3, i}^{-1} \hat{T}_{4, i} \hat{T}_{2, i}, & \hat{T}_{3, i}^{-1}-\hat{T}_{3, i}^{-1} \hat{T}_{4, i} \hat{T}_{1, i}
\end{array}\right) .
$$

If there are $L$ columns in the $x$ direction, the total transfer matrix which gives the relation between amplitudes at two end columns is

$$
\hat{T}=\prod_{i=1}^{L} \hat{T}_{L-i+1}
$$

If we want to investigate the propagation properties in the $y$ direction, we have to establish the transfer matrix for rows. The relation between two adjacent rows is written as

$$
\left(\begin{array}{c}
\mathbf{v}_{j+1,-1} \\
\mathbf{v}_{j+1,1}
\end{array}\right)=\hat{V}_{j}\left(\begin{array}{c}
\mathbf{v}_{j,-1} \\
\mathbf{v}_{j, 1}
\end{array}\right),
$$

where $\mathbf{v}_{j, l}$ 's are vectors denoting rows with length $M, \mathbf{v}_{j, l} \equiv\left(c_{1, j, l}, c_{2, j, l}, \ldots, c_{M, j, l}\right)^{T}$, and the elements of the transfer matrix are

$$
\left\{\hat{V}_{j}\right\}_{i, l ; i^{\prime}, l^{\prime}}=\delta_{l,-(-1)^{i}}\left\{C_{i, j}^{(1)} \delta_{-l, l^{\prime}} \delta_{i, i^{\prime}}-C_{i, j}^{(2)} \delta_{l, l^{\prime}} \delta_{i, i^{\prime}}-C_{i, j}^{(3)} \delta_{l, l^{\prime}} \delta_{i+(-1)^{i}, i^{\prime}}\right.
$$




$$
\begin{array}{r}
+C_{i, j}^{(4)}\left(C_{i, j}^{(1)} \delta_{l, l^{\prime}} \delta_{i, i^{\prime}}-C_{i, j}^{(2)} \delta_{l,-l^{\prime}} \delta_{i, i^{\prime}}-C_{i, j}^{(3)} \delta_{l,-l^{\prime}} \delta_{i+(-1)^{i}, i^{\prime}}\right)-C^{(5)} \delta_{l, l^{\prime}} \delta_{l,-(-1)^{i}} \delta_{i, i^{\prime}} \\
\left.-C^{(6)}\left(C_{i-(-1)^{i}, j^{\prime}}^{(1)} \delta_{l,-l^{\prime}} \delta_{i-(-1)^{i}, i^{\prime}}-C_{i-(-1)^{i}, j}^{(2)} \delta_{l, l^{\prime}} \delta_{i-(-1)^{i}, i^{\prime}}-C_{i-(-1)^{i}, j^{i}}^{(3)} \delta_{l, l^{\prime}} \delta_{i-(-1)^{i} \cdot 2, i^{\prime}}\right)\right\},
\end{array}
$$

where

$$
\begin{gathered}
C_{i, j}^{(1)}=\frac{E-\epsilon_{i, j,(-1)^{i}}}{t_{i, j,(-1)^{i} ; i, j+1,-(-1)^{i}}}, \quad C_{i, j}^{(2)}=\frac{t_{i, j,(-1)^{i} ; i, j,-(-1)^{i}}}{t_{i, j,(-1)^{i} ; i, j+1,-(-1)^{i}}}, \quad C_{i, j}^{(3)}=\frac{t_{i, j,(-1)^{i} ; i+(-1)^{i}, j,-(-1)^{i}}}{t_{i, j,(-1)^{i} ; i, j+1,-(-1)^{i}}}, \\
C_{i, j}^{(4)}=\frac{E-\epsilon_{i, j+1,-(-1)^{i}}}{t_{i, j+1,-(-1)^{i} ; i, j+1,(-1)^{i}}}, \quad C_{i, j}^{(5)}=\frac{t_{i, j+1,-(-1)^{i} ; i, j,(-1)^{i}}}{t_{i, j+1,-(-1)^{i} ; i, j+1,(-1)^{i}}}, \quad C_{i, j}^{(6)}=\frac{t_{i, j+1,-(-1)^{i} ; i-(-1)^{i}, j+1,(-1)^{i}}}{t_{i, j+1,-(-1)^{i} ; i, j+1,(-1)^{i}}} .
\end{gathered}
$$

The total transfer matrix for $L$ rows is

$$
\hat{V}=\prod_{i=1}^{L} \hat{V}_{L-i+1} .
$$

For a finite $M \times L$ system, there are $M$ propagating channels along the length $L$. The Hermitian matrix, $\left(\hat{T}^{\dagger} \hat{T}\right)^{1 / 2 L}$ or $\left(\hat{V}^{\dagger} \hat{V}\right)^{1 / 2 L}$, has $2 M$ eigenvalues. These $2 M$ eigenvalues should be positive and come in inverse pairs due to the unitarity of the matrix [37, 38, 39, 40]. The $M$ positive logarithms of eigenvalues, denoted as $\gamma_{i}$ in an ascending order with index $i$, are Lyapunov exponents (LE) reflecting the exponential decay of the corresponding channels. Obviously, the logarithms of other $M$ eigenvalues are the negatives of $\gamma_{i}$. The LEs will approach 0 when the disorder vanishes. The localization length is defined as the reciprocal of the smallest $\operatorname{LE} \xi \equiv 1 / \gamma_{1}$. The localization length plays a crucial role in the localization theory.

The zero-temperature conductance $g$ of a rectangular $M \times L$ system in units of $2 e^{2} / h$, with two spins being taken into account, can be evaluated using $M$-channel Landauer formula [41, 42, 43]

$$
g=\operatorname{Tr}\left(\hat{t}^{\dagger} \hat{t}\right)
$$

where the $M \times M$ transmission matrix $\hat{t}$ describes the transmission of electrons from one lead to the other in the longitudinal direction. Here we assume that the leads are connected to all the $M$ channels and they are perfect metals with band width much larger than that of graphene. Formula (20) can be expressed with the LEs as [37, 40]

$$
g=\sum_{i=1}^{M} \frac{1}{\cosh ^{2}\left(\gamma_{i} L\right)} .
$$

Numerically, LEs can be calculated by using the standard method of Gram-Schmidt re-orthonormalization after each a few, say, ten steps of multiplication of transfer matrices 
[44, 45]. This is equivalent to the diagonalization of $\hat{T}^{\dagger} \hat{T}$ or $\hat{V}^{\dagger} \hat{V}$, but avoids terrible overflow and loss of precision on the computer. On the other hand, although the calculations of transfer matrices along the $x$ and $y$ directions are certainly different, there is no essential difference in the calculated results between these two directions. This is owing to the fact that the dispersion relation is conic around the Dirac point which is isotropic in the $x$ and $y$ directions. Below we will only present the results obtained for the transmission along the $y$ direction.

\section{LOCALIZATION IN SYSTEM WITH DIAGONAL DISORDER}

First we investigate the case of diagonal disorder, i.e., $W \neq 0, \lambda=0$, and $s=0$. The lowest LE $\gamma_{1}(M)$ is calculated for very long $\left(L \sim 10^{6}\right)$ strip of width $M$. The rescaled localization length is defined as $\frac{\Lambda(M)}{M}=\frac{1}{\gamma_{1}(M) M}$. For given energy and disorder strength, the localization behavior of the fermions can be determined by the scaling dependence of $\frac{\Lambda(M)}{M}$ : If $\frac{\Lambda(M)}{M}$ increases with $M$, the states are extended. For localized states $\frac{\Lambda(M)}{M}$ is decreased by increasing $M$. The $M$-independence of $\frac{\Lambda(M)}{M}$ corresponds to the fixed point separating regions of extended and localized states. In Fig. 2(a), we plot the $M$ dependence of $\frac{\Lambda(M)}{M}$ for states at $E=0$ in systems with different strengths of diagonal disorder. For all investigated strengths of diagonal disorder, the rescaled localization length always decreases with increasing width. The large fluctuations in the curve of $W=1$ are due to numerical errors in the Gram-Schmidt re-orthonormalization procedure which become serious in the case with $\frac{\Lambda(M)}{M} \gg 1$. This means that the states at the Dirac point are localized by tiny diagonal disorder. In Fig. 2(b), we plot the $M$-dependence of $\frac{\Lambda(M)}{M}$ for a given strength of diagonal disorder but different energies. Although all states are localized, the scaling behavior is much different between the states at Dirac point and the other states: the decreasing slope of the former is much larger than that of the latter. This implies that the states at Dirac point are more easily localized than other states by introducing the diagonal disorder in the thermodynamical limit, in spite of their larger localization length in smaller systems. This may be due to the small density of states at $E=0$ and the breaking of the chiral symmetry by diagonal disorder. The localization behavior of the states is consistent with the common theories of the Anderson localization in 2D. 

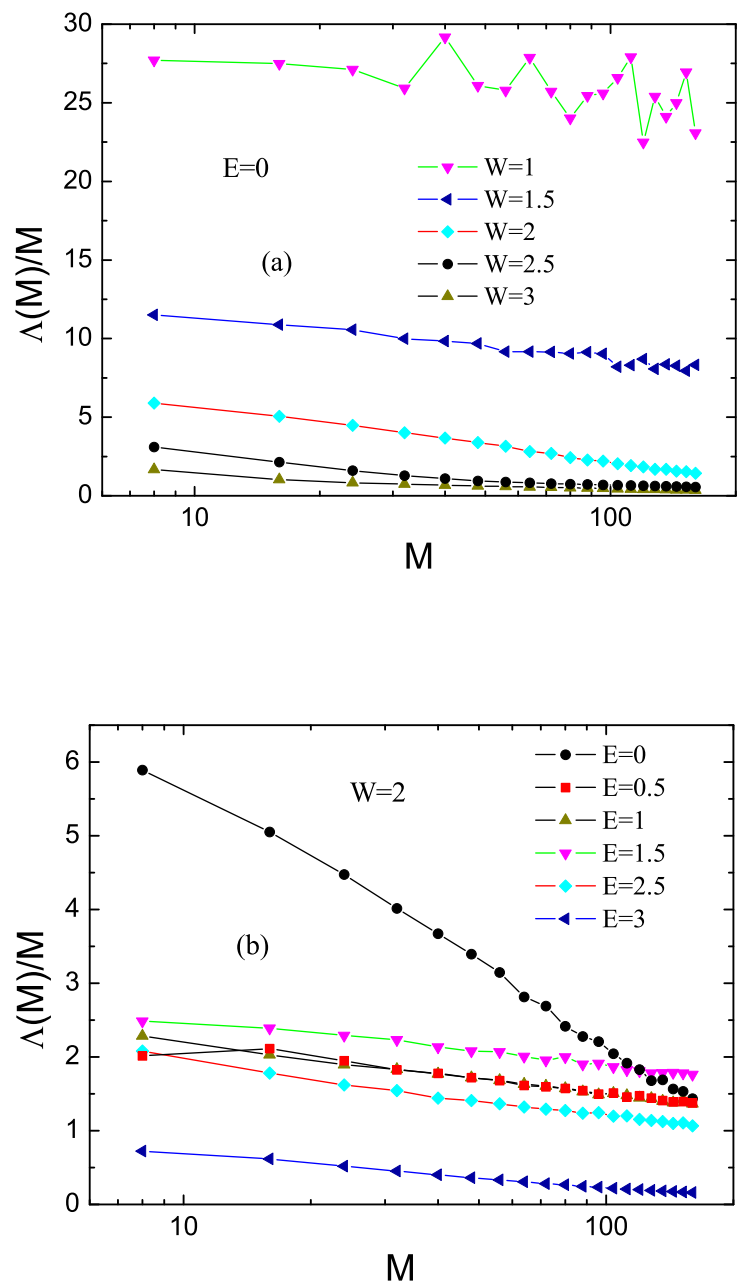

FIG. 2: (Color online) Scaling behavior of rescaled localization length in the presence of diagonal disorder. (a) $\frac{\Lambda(M)}{M}$ versus $M$ at the Dirac point $(E=0)$ for different strengths of disorder. (b) $\frac{\Lambda(M)}{M}$ versus $M$ for a given strength of disorder and various energies.

\section{DELOCALIZATION AT DIRAC POINT IN THE CASE OF OFF-DIAGONAL DISORDER WITHOUT SIGN CHANGES}

Differently from diagonal disorder, off-diagonal disorder does not break the chiral symmetry at Dirac point, leading to an essential difference in the localization. The scaling analysis of the rescaled localization length and the conductance at Dirac point is shown in Fig. 3 by using the distribution function $P_{o 1}$ in Eq. (3). This corresponds to the case of randomly warped or corrugated lattice where bond lengths are randomly shifted from the perfect value. 
From Fig. 3(a) we can see that the rescaled localization length is independent of $M$ for all disorder strengths. This implies that the localization length at the thermodynamical limit is infinite, corresponding to delocalized states at $E=0$. The conductance of an $M \times M$ system calculated from Eq. (21) is also independent of $M$ for all investigated strengths of off-diagonal disorder [see Fig. 3(b)]. So the conductance at the thermodynamical limit can be determined from these size independent values. The dependence of $g$ on the disorder strength is shown in the inset of Fig. 3(b). For relatively weak off-diagonal disorder $(\lambda<1)$ the conductance at Dirac point is about $\frac{4 e^{2}}{h}$, corresponding to the quantum conductance of 4 channels ( 2 spin channels and 2 equivalent channels from the chiral symmetry at $E=0$ ). In fact, in a system with off-diagonal disorder, the calculated Lyapunov exponents at $E=0$ can be grouped into pairs, i.e., $\gamma_{2 i-1}=\gamma_{2 i}$ for integer $i$ in the range between 1 and $\frac{M}{2}$, corresponding to two equivalent channels with the chiral transformation. These channels have the localization length proportional to the width $M$. At the thermodynamical limit they become infinite. By increasing the disorder strength from $\lambda=1$ towards its maximum value $\lambda=2$, these channels are still delocalized at the thermodynamical limit but the conductance rapidly drops, as can be seen from the inset of Fig. 3(b).

The delocalization behavior at $E=0$ in graphene with the off-diagonal disorder is not consistent with the usual localization behavior of 2D disordered systems. One may attribute this inconsistency to the chiral symmetry at $E=0$ which is conserved in off-diagonal disorder but destroyed in diagonal disorder. So the localization at energies $E \neq 0$, where the chiral symmetry no longer exists, could be expected. In Fig. 4, we plot the scaling behavior of the rescaled localization length and the conductance of an $M \times M$ system for different energies. Except for the curves of $E=0$, both the rescaled localization length and the conductance decrease by increasing $M$ in the general trend if fluctuations of some curves are ignored, suggesting the usual localization behavior at $E \neq 0$. From Fig. 4(a), the rescaled localization length is generally decreased by increasing $E$ from $E=0$ for a fixed $M$. This means that in off-diagonal disorder the states of energies farther apart from Dirac point are more localized. From Fig. 4(b), however, for a given value of $M$ the conductance at larger $|E|$ has larger values. This can be explained by the fact that there are more electron channels at larger $|E|$ in graphene which can contribute to the conductance if $M$ is not very large.

By using a scaling transformation $M \rightarrow M / \xi$ with appropriate value of scaling factor $\xi$ 

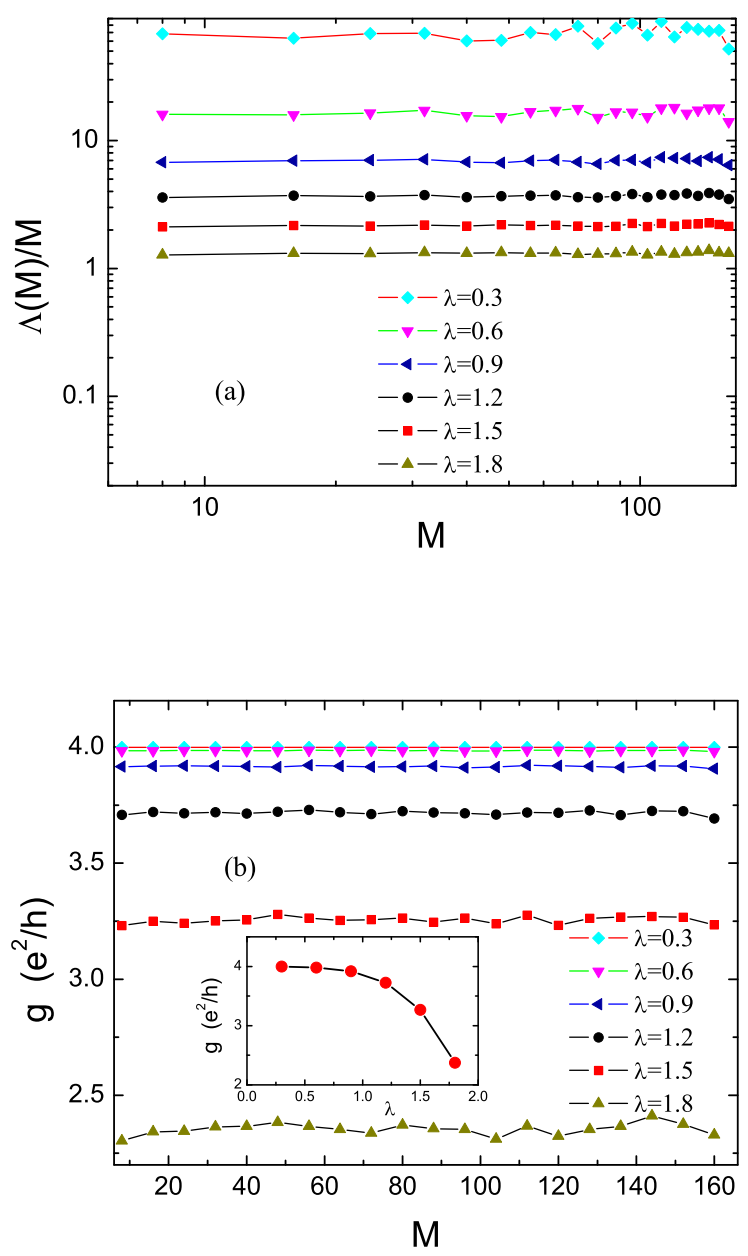

FIG. 3: (Color online) Scaling behavior of the rescaled localization length (a) and the conductance of an $M \times M$ system (b) at the Dirac point $(E=0)$ in the presence of off-diagonal disorder. The inset of (b) shows the dependence of $g$ on the strength of off-diagonal disorder $\lambda$.

for every energy $E$, the curves in Fig. 4(a) for different energies can merge together. In Fig. 5 we plot the common dependence of $\frac{\Lambda(M)}{M}$ on $M / \xi$ for various energies. The scaling factor $\xi$ as a function of $E$ is shown in the inset which can be fitted by a simple function $\xi=\frac{a}{|E|}$ with prefactor $a=2.66$. Since $\xi$ is just the localization length at the thermodynamical limit, this scaling behavior further ensures the delocalization at $E=0$ and describes how the localization length diverges when $E \rightarrow 0$. From the Dirac dispersion relation the wave length of electrons is also inversely proportional to $|E|$. This suggests that in the case of off-diagonal disorder the localization length and the electron wave length have the same $E$ 

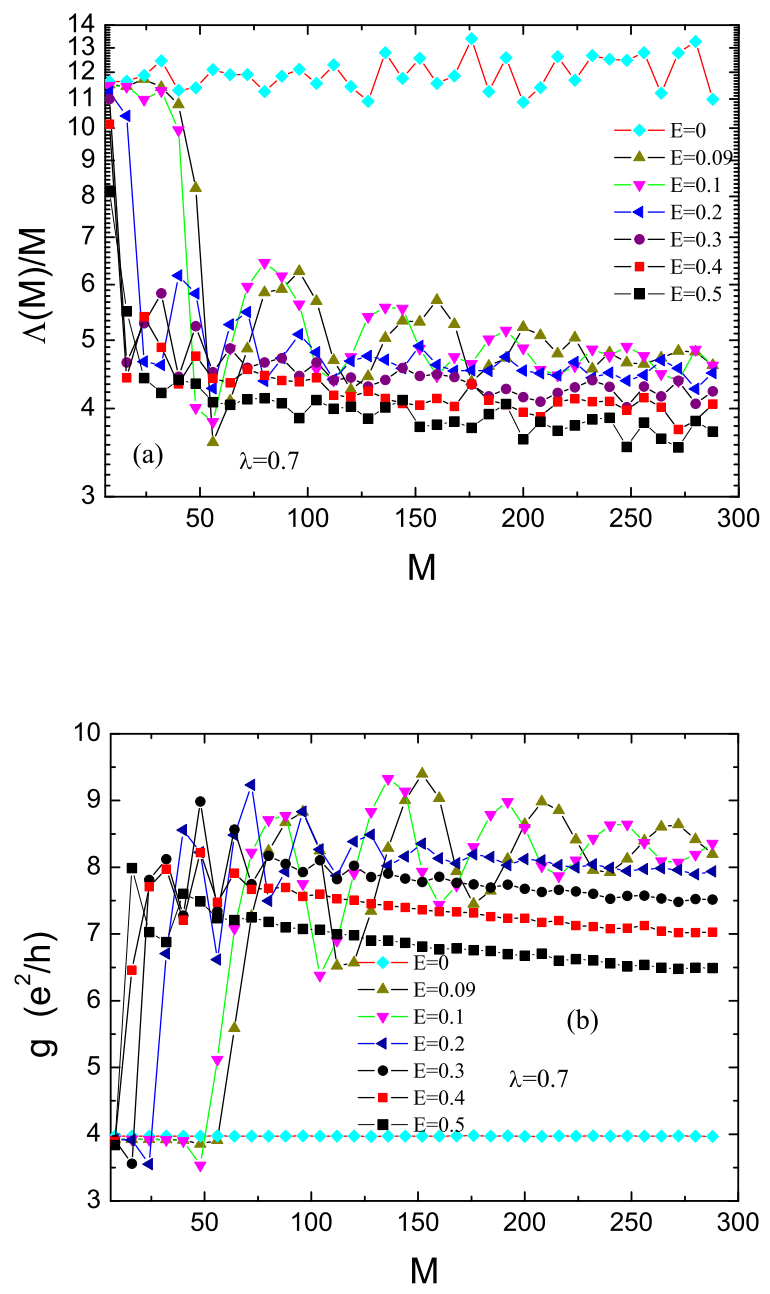

FIG. 4: (Color online) Scaling behavior of the rescaled localization length (a) and the conductance of an $M \times M$ system (b) for different energies in the presence of off-diagonal disorder.

dependence and can be regarded as the same length scale. We also notice that although the states at $E=0$ are delocalized, there is no real metallic region with finite range of $E$. This is certainly different from the results in Refs. 29, 30, 31, 32, 33 where the disorder models free of intervalley scattering are adopted.

The different localization behaviors between $E=0$ and $E \neq 0$ can be more clearly depicted by the spatial distributions of wavefunctions as shown in Fig. 6. As can be seen from Fig. 6(a), the delocalized state at $E=0$ is more likely to be a Cantor-set like critical state, rather than a real metallic state. Such states may provide propagation paths for current from one edge to the other. On the other hand, the state at $E=1.8$ shown in 


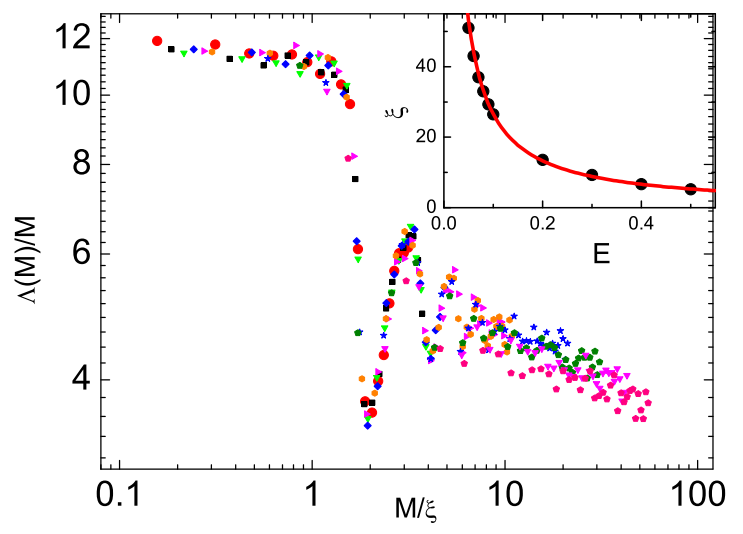

FIG. 5: (Color online) Common dependence of the rescaled localization length $\frac{\Lambda}{M}$ on the scaled width $\frac{M}{\xi}$ for various energies. Inset: $\xi$ as a function of $E$ (circle symbols) which is fitted by function $\xi=\frac{2.66}{E}$ (red curve).

Fig. 6(b) has significant amplitudes only in some isolated islands, corresponding to typical localization.

In the case of off-diagonal disorder there are 4 delocalized channels at Dirac point, giving conductance $\frac{4 e^{2}}{h}$. This leads to almost shape-independent conductance and shape-dependent conductivity. The situation is similar to the case of perfect lattice investigated by Tworzydlo et al. in Ref. 46. Following the procedure proposed in Ref. 46, we calculate the conductivity from the conductance of a $M \times L$ rectangular sample. The obtained shape dependence of the conductivity is shown in Fig. 7. This behavior is consistent with the nature of the quantum transport in $2 \mathrm{D}$, but is inconsistent with the experiments where the conductivity is shape independent. This means that to explain this experimental result we have to invoke some mechanism which could convert the quantum transport to the classical-like transport.

\section{CLASSICAL-LIKE TRANSPORT AT DIRAC POINT IN THE CASE OF OFF- DIAGONAL DISORDER WITH SIGN CHANGES}

The quantum transport processes could be drastically changed if the phases of wavefunctions become uncertain. This may happen due to the geometric phase which can be acquired by electrons during a cyclic evolution of "slow" degrees of freedom around a conic point. 

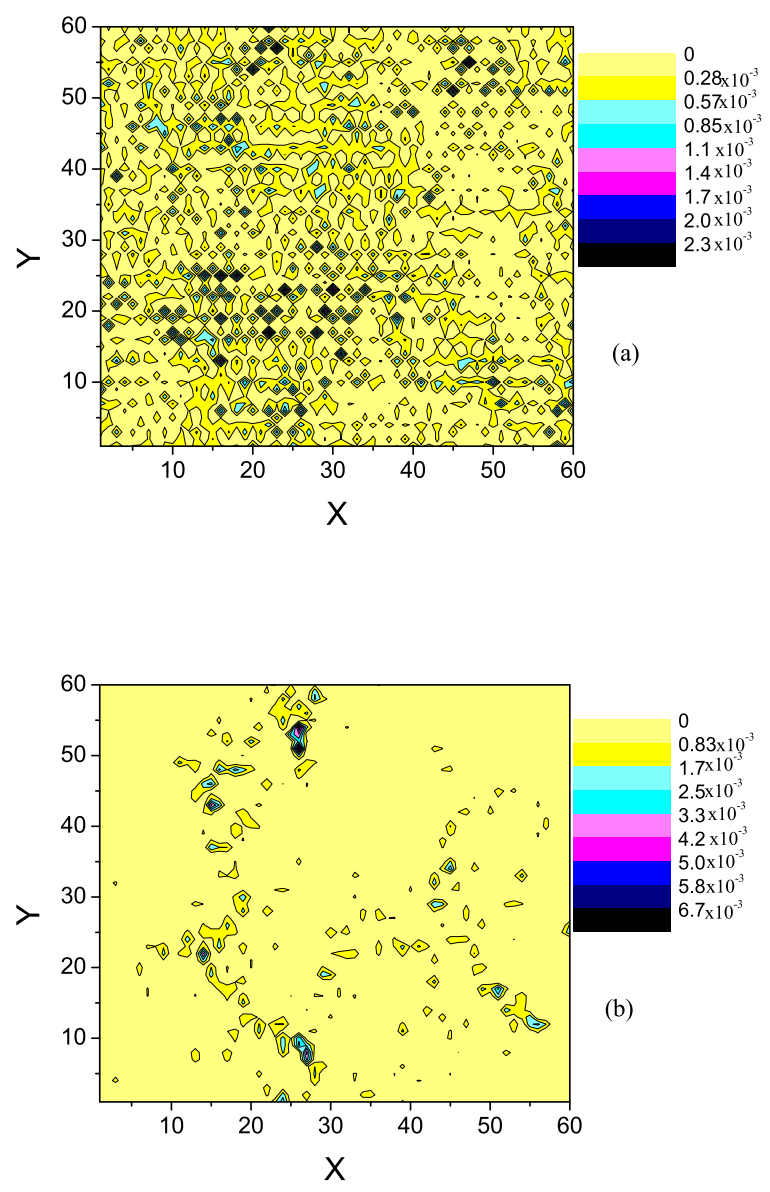

FIG. 6: (Color online) Contour plot of spatial distributions of wavefunctions at (a) $E=0$ and (b) $E=1.8$ in a $60 \times 60$ system in off-diagonal disorder of strength $\lambda=0.7$.

The additional geometric phase of $\sim \pm \pi$ corresponds to sign changes of the wavefunction during the motion of tunneling electrons. In the diffusion regime, if the sign changes randomly happen during the spatial motion, they can be modeled by a random distribution of positive and negative signs of hopping integrals. In probability $P_{o}\left(t_{n n^{\prime}}\right)$ of Eq. (4) we include both the sign randomness and the magnitude randomness of the hopping integrals which are specified by parameters $s$ and $\lambda$, respectively.

First we set $\lambda=0$ and focus on the effect of sign randomness. In Fig. 8 we plot the scaling behavior of the rescaled localization length and the conductance of an $M \times M$ system for different values of $s$. Similarly to the case of off-diagonal disorder, the states at the Dirac point in the presence of the sign randomness are also delocalized in the sense of finite-size 


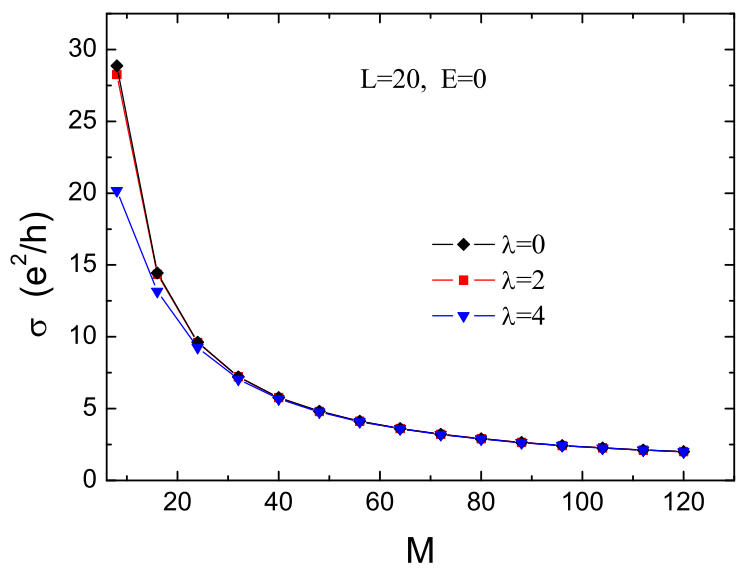

FIG. 7: (Color online) Width dependence of conductivity of an $M \times L$ rectangular system with a given length at $E=0$ for different strengths of off-diagonal disorder.

scaling analysis. Differently from the behavior in the off-diagonal disorder shown in Fig. 3, effects of sign randomness on the rescaled localization length and on the conductance are opposite: $\frac{\Lambda(M)}{M}$ in $s=0.5$ is smaller than that in $s=0.2$, but $g$ in $s=0.5$ is larger than that in $s=0.2$. This is strange because $\frac{\Lambda(M)}{M}$ corresponds to the channel with the largest localization length which usually provides the leading contribution to the conductance. The only explanation for this is that the other channels also give significant contributions in the case of sign randomness. An expectable consequence of this is a new shape dependence of the conductivity, since the number of the tunneling channels is no longer restricted.

The shape dependence of the conductivity can be investigated by calculating the conductance of $M \times L$ rectangular samples. The obtained conductivity as a function of ratio $M / L$ for various sizes is shown in Fig. 9. Except for small values of $M / L$, the conductivity has values near $\frac{4 e^{2}}{h}$, independent of $M / L$ and $M$, in consistence with the experimental findings. Surprisingly, this value is even larger than $\frac{4 e^{2}}{\pi h}$ obtained in ballistic graphene [46, 47] in spite of the disorder introduced by the sign randomness of hopping integrals. To get insight into this anomalous feature, we adopt the average spacing between successive pairs of Lyapunov exponents denoted as $\delta \gamma$, i.e., $\gamma_{2 i+1}-\gamma_{2 i-1} \sim \gamma_{2 i+2}-\gamma_{2 i} \sim \delta \gamma$. Notice that in this case the Lyapunov exponents are grouped into pairs, corresponding to two equivalent channels from the chiral symmetry. By keeping only the leading exponential term of $\cosh ^{2}\left(\gamma_{i} L\right)$, Eq. (21) 


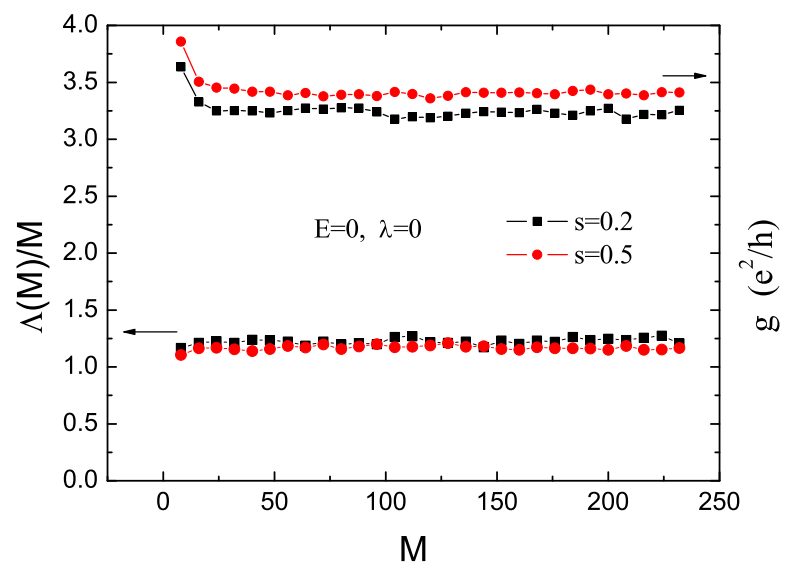

FIG. 8: (Color online) Scaling behavior of the rescaled localization length and the conductance of an $M \times M$ system in the presence of sign randomness of hopping integrals obeying probability $P_{o}\left(t_{n n^{\prime}}\right)$ with different values of $s$.

can be approximately rewritten as

$$
g \approx \sum_{i=1}^{M / 2} 8 e^{-2 \gamma_{2 i-1} L} \approx \frac{8 e^{-2 \gamma_{1} L}\left(1-e^{-(M+2) L \delta \gamma}\right)}{1-e^{-2 L \delta \gamma}},
$$

in units of $\frac{2 e^{2}}{h}$. In a perfect system of width $M$ and using the periodic boundary condition, the spacing $\delta \gamma$ for states at $E=0$ is $\delta \gamma=2 \pi / M$ due to the Dirac fermion dispersion relation [47]. One may reasonably suppose that $\delta \gamma \propto 1 / M$ is still held in the case of OD disorder. Then, for $M \gg L$, the conductivity of an $M \times L$ rectangular sample can be calculated as

$$
\sigma=\frac{g L}{M} \approx \frac{4 e^{-2 \gamma_{1} L}}{\delta \gamma M}
$$

So for the perfect lattice $\sigma=\frac{2}{\pi}$ in units of $\frac{2 e^{2}}{h}$ [46, 47]. In the case of sign randomness, the values of $M \delta \gamma$ are in the range $[0.9,2.5]$ as shown in the inset of Fig. 9. Together with the factor $e^{-2 \gamma_{1} L}$, the obtained conductivity may be $\pi$ times of that of the perfect lattice. Thus, the $\pi$ factor difference in the conductivity is not trivial and reflects an essential change of the fermion properties due to the phase uncertainty.

It is interesting to investigate how the localization behavior changes in the presence of the magnitude randomness of hopping integrals. The scaling behaviors of the rescaled localization length and the conductance of an $M \times M$ system with both nonzero $s$ and $\lambda$ are shown in Fig. 10. It can be seen that the states at $E=0$ are still delocalized and 


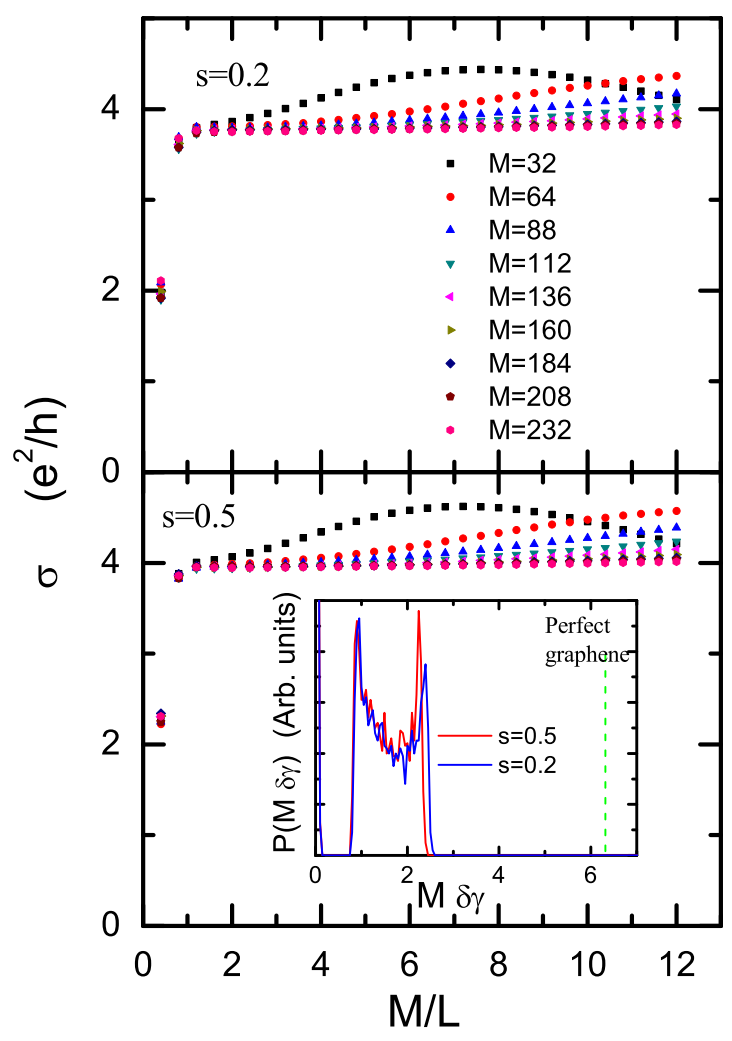

FIG. 9: (Color online) Calculated conductivity as a function of ratio $M / L$ for rectangular sheets with different widths $M$. Inset in the lower panel: Distribution function $P(M \delta \gamma)$ of $M \delta \gamma$. The green dashed line indicates the $\delta$-function distribution at $2 \pi$ in the case of perfect sheet.

the opposite effects on the rescaled localization length and on the conductance are more evident than those shown in Fig. 8. In Fig. 11 we display the shape dependence of the conductivity of the $M \times L$ rectangular samples for $s=0.5$ and different nonzero values of $\lambda$. Similarly to the case of $\lambda=0$, the conductivity is still shape independent except for small values of $M / L$. This means that the sign-randomness induced shape independence of the conductivity is robust against the warping or corrugation disorder which causes randomness of magnitudes of hopping integrals. It can also be seen that the value of the conductivity is slightly reduced from $\frac{4 e^{2}}{h}$ by increasing the warping or corrugation disorder. For reasonable strength of the warping disorder $(\lambda<1)$, however, the conductivity is above $\frac{3 e^{2}}{h}$, in the range of measured values in experiments.

It is also interesting to investigate the scaling behavior of the statistics of the minimum 


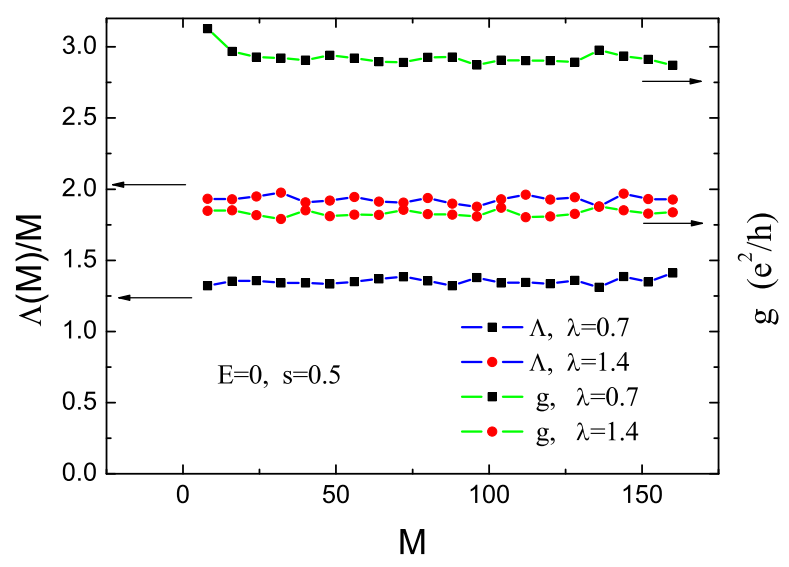

FIG. 10: (Color online) Scaling behavior of the rescaled localization length and the conductance of an $M \times M$ system in the presence of both sign randomness and magnitude randomness of hopping integrals obeying probability $P_{o}\left(t_{n n^{\prime}}\right)$.

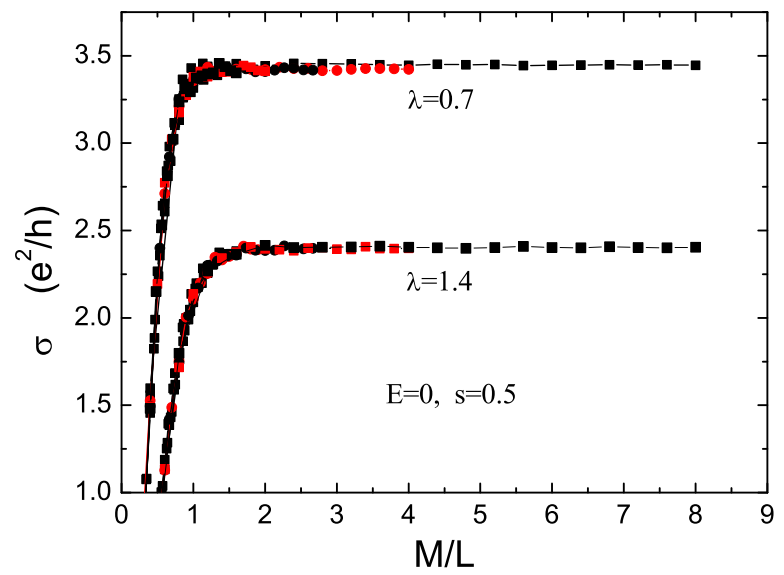

FIG. 11: (Color online) Calculated conductivity as a function of ratio $W / L$ for rectangular sheets with different widths $M$ in the presence of both sign randomness and magnitude randomness of hopping integrals.

conductivity. In Fig. 12 we plot the statistical distribution of the conductivity at $E=0$ for various system sizes and for given values of $M / L, \lambda$, and $s$. We note that the distribution of the conductivity is Gaussian-like, and both the average and the distribution width are 


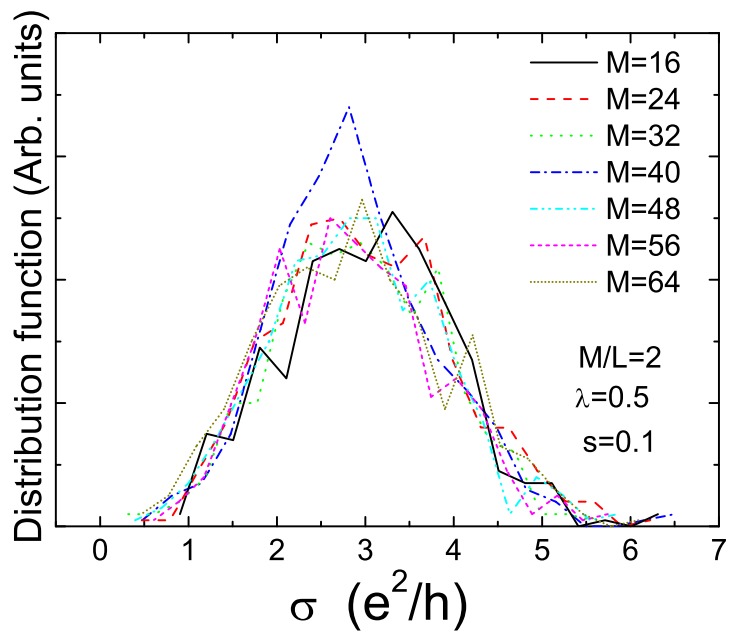

FIG. 12: (Color online) Statistical distribution of the conductivity at $E=0$ for various system sizes in OD disorder with sign randomness. The values of $M / L, \lambda$, and $s$ are given in the figure. The statistics is taken from 500 random configurations.

almost scaling invariant. This is different from the statistics of a single Lyapunov exponent in usual disordered system for which the distribution width is $1 / M$ dependent. This difference originates from the fact that in the present system the conductivity is contributed not only from the channel with the smallest LE but also from other channels. As a consequence the statistics of the conductivity given by Eq. (23) is mainly determined by the distribution of $\delta \gamma M$ rather than by the distribution of a single LE. It can be easily verified that in the present case the series of successional LEs times $M, \gamma_{1} M, \gamma_{2} M, \ldots$, are scaling invariant. Thus the statistics of $\delta \gamma M$ is also scaling invariant, leading to the scaling invariant statistics of the minimum conductivity.

\section{CONCLUSIONS}

We investigate the effect of different types of disorder on the transport properties of electrons at Dirac point in graphene. From the finite-size scaling analysis together with the transfer-matrix technique, it is found that all states are localized in the presence of the diagonal disorder, in consistence with the main conclusions of the scaling theory for $2 \mathrm{D}$. In the case of off-diagonal disorder, however, the states at $E=0$ are delocalized. This delocaliza- 
tion, inconsistent with the scaling theory and only existing at Dirac point $(E=0)$, is mainly due to the specific chiral symmetry which is conserved in off-diagonal disorder but destroyed by diagonal disorder. The obtained conductance at the thermodynamical limit shows two transparent chirally symmetric quantum channels at $E=0$. These channels, however, could not produce shape independent conductivity observed in experiments. It can be obtained only by introducing the sign randomness of hopping integrals. We investigate the scaling behavior of the rescaled localization length and the conductance in the presence of both sign randomness and magnitude randomness to show the existence of delocalized states at $E=0$. Especially, the calculated conductivity of a rectangular sample is shape independent. As the sign randomness of the hopping integrals corresponds to some additional uncertain phases, the obtained results reflect a general trend of transition from quantum to classical transport in dephasing processes. Although the calculated conductivity is in consistence with the experimental findings, the mechanism for the phase uncertainty still needs to be identified by future investigations.

\section{Acknowledgments}

This work was supported by the State Key Programs for Basic Research of China (2005CB623605 and 2006CB921803), and by National Foundation of Natural Science in China Grant Nos. 10474033, 10704040, and 60676056.

[1] K. S. Novoselov, A. K. Geim, S. V. Morozov, D. Jiang, Y. Zhang, S. V. Dubonos, I. V. Grigorieva, and A. A. Firsov, Science 306, 666 (2004).

[2] C. Berger, Z. Song, T. Li, X. Li, A. Y. Ogbazghi, R. Feng, Z. Dai, A. N. Marchenkov, E. H. Conrad, P. N. First, and W. A. de Heer, J. Phys. Chem. B 108, 19912 (2004).

[3] K. S. Novoselov, A. K. Geim, S. V. Morozov, D. Jiang, M. I. Katsnelson, I. V. Grigorieva, S. V. Dubonos, and A. A. Firsov, Nature (London) 438, 197 (2005).

[4] Y. Zhang, Y.-W. Tan, H. L. Stormer, and P. Kim, Nature (London) 438, 201 (2005).

[5] C. Berger, Z. Song, X. Li, X. Wu, N. Brown, C. Naud, D. Mayou, T. Li, J. Hass, A. N. Marchenkov, E. H. Conrad, P. N. First, and W. A. de Heer, Science 312, 1191 (2006). 
[6] K. S. Novoselov, A. K. Geim, S. V. Morozov, D. Jiang, M. I. Katsnelson, I. V. Grigorieva, S. V. Dubonos, and A. A. Firsov, Nature 438, 197 (2005).

[7] Y. Zhang, J. P. Small, M. E. S. Amori, and P. Kim, Phys. Rev. Lett. 94, 176803 (2005).

[8] J. C. Slonczewski and P. R. Weiss, Phys. Rev. 109, 272 (1958).

[9] D. DiVincenzo and E. Mele, Phys. Rev. B 29, 1685 (1984).

[10] E. Abrahams, P. W. Anderson, D. C. Licciardello, and T.V. Ramakrishnan, Phys. Rev. Lett. 42, 673 (1979).

[11] P. A. Lee, Phys. Rev. Lett. 71, 1887 (1993).

[12] A. W. W. Ludwig, M. P. A. Fisher, R. Shankar, and G. Grinstein, Phys. Rev. B 50, 7526 (1994).

[13] A. A. Nersesyan, A. M. Tsvelik, and F.Wenger, Phys. Rev. Lett. 72, 2628 (1994).

[14] K. Ziegler, Phys. Rev. Lett. 80, 3113 (1998).

[15] N. M. R. Peres, F. Guinea, A. H. Castro Neto, Phys. Rev. B 73, 125411 (2006).

[16] S.V. Morozov, K. S. Novoselov, M. I. Katsnelson, F. Schedin, L. A. Ponomarenko, D. Jiang, and A. K. Geim, Phys. Rev. Lett. 97, 016801 (2006).

[17] E. Fradkin, Phys. Rev. B 33, 3257 (1986).

[18] N. H. Shon and T. Ando, J. Phys. Soc. Jpn. 67, 2421 (1998); H. Suzuura and T. Ando, Phys. Rev. Lett. 89, 266603 (2002).

[19] V. M. Pereira, F. Guinea, J. M. B. L. dos Santos, N. M. R. Peres, and A. H. C. Neto, Phys. Rev. Lett. 96, 036801 (2006).

[20] D.V. Khveshchenko, Phys. Rev. Lett. 97, 036802 (2006).

[21] E. McCann, K. Kechedzhi, V. I. Fal'ko, H. Suzuura, T. Ando, and B. L. Altshuler, Phys. Rev. Lett. 97, 146805 (2006).

[22] A. F. Morpurgo and F. Guinea, Phys. Rev. Lett. 97, 196804 (2006).

[23] I. L. Aleiner and K. B. Efetov, Phys. Rev. Lett. 97, 236801 (2006).

[24] A. Altland, Phys. Rev. Lett. 97, 236802 (2006).

[25] K. Ziegler, Phys. Rev. Lett. 97, 268802 (2006).

[26] K. Nomura and A. H. MacDonald, Phys. Rev. Lett. 98, 076602 (2007).

[27] D. N. Sheng, L. Sheng, and Z. Y. Weng, Phys. Rev. B 73, 233406 (2006).

[28] E. Louis, J. A. Vergés, F. Guinea, and G. Chiappe, Phys. Rev. B 75, 085440 (2007).

[29] P. M. Ostrovsky, I.V. Gornyi, and A. D. Mirlin, Phys. Rev. Lett. 98, 256801 (2007). 
[30] M. Titov, Europhys. Lett. 79, 17004 (2007).

[31] J. H. Bardarson, J. Tworzydlo, P. W. Brouwer, and C. W. J. Beenakker, arXiv: condmat/0705.0886.

[32] K. Nomura, M. Koshino, and S. Ryu, arXiv: cond-mat/0705.1607.

[33] P. San-Jose, E. Prada, D. S. Golubev, arXiv: cond-mat/0706.3832.

[34] T. A. L. Ziman, Phys. Rev. B 26, 7066 (1981).

[35] R. Gade and F. Wegner, Nucl. Phys. B 360, 213 (1991).

[36] Shi-Jie Xiong and S. N. Evangelou, Phys. Rev. B 64, 113107 (2001).

[37] B. Kramer and A. MacKinnon, Rep. Progr. Phys. 56, 1469 (1993).

[38] V. I. Oseledets, Trans. Moscow Math. Soc. 19, 197 (1968).

[39] K. Ishii, Suppl. Progr. Theor. Phys. 53, 7 (1973).

[40] C. W. J. Beenakker, Rev. Mod. Phys. 69, 731 (1997).

[41] R. Landauer, Philos. Mag. 21, 863 (1970).

[42] D. S. Fisher and P. A. Lee, Phys. Rev. B 23, R6851 (1981).

[43] E. N. Economou and C. M. Soukoulis, Phys. Rev. Lett. 46, 618 (1981).

[44] A. MacKinnon and B. Kramer, Phys. Rev. Lett. 47, 1546 (1981).

[45] A. MacKinnon and B. Kramer, Z. Phys. B 53, 1 (1983).

[46] J. Tworzydlo, B. Trauzettel, M. Titov, A. Rycerz, and C.W. J. Beenakker, Phys. Rev. Lett. 96, $246802(2006)$.

[47] M. I. Katsnelsona, Eur. Phys. J. B 51, 157 (2006). 